\title{
CARACTERIZAÇÃO FÍSICA DE SUBSTRATOS À BASE DE SERRAGEM E RECIPIENTES PARA CRESCIMENTO DE MUDAS DE CACAUEIRO ${ }^{1}$
}

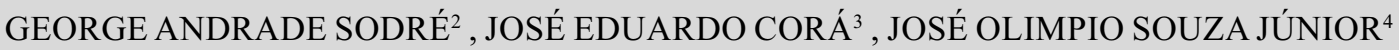

RESUMO - O objetivo deste trabalho foi realizar caracterização física e avaliar o efeito de substratos à base de serragem e dois recipientes no crescimento de mudas de cacaueiro. O delineamento experimental foi em blocos casualizados, em esquema fatorial $2 \mathrm{x}$ 4 x 2. Os tratamentos foram obtidos da combinação de serragens originadas de dois locais: municípios de Una e Camacan, no Estado da Bahia, quatro proporções (v:v) de serragem e areia: 1:0; 8:1; 4:1 e 2:1 e dois recipientes de crescimento (tubetes de $288 \mathrm{~cm}^{3}$ e sacos de polietileno de $840 \mathrm{~cm}^{3}$ ). Antes do plantio, amostras dos substratos foram retiradas para análises físicas. Foram usadas miniestacas de 4 a $6 \mathrm{~cm}$ de comprimento do clone Trinidad Select Hybrid (TSH 1188). Inicialmente, as miniestacas foram tratadas na base com AIB $6.000 \mathrm{mg} \mathrm{kg}^{-1}$, em seguida foram inseridas em tubetes preenchidos com os substratos e mantidas em câmara de nebulização. Após quarenta dias, as miniestacas foram retiradas da câmara e transferidas para crescimento, em casa de vegetação, onde parte foi mantida nos respectivos tubetes e outra transplantada em sacos de polietileno de $840 \mathrm{~cm}^{3}$ preenchidos com os mesmos tratamentos. Após cinco meses, em casa de vegetação, as mudas foram avaliadas quanto à altura da planta, diâmetro do caule, massa da matéria seca da parte aérea e das raízes, número de folhas e área foliar das plantas. Na análise física dos substratos, verificou-se que a distribuição do tamanho de partículas foi diferenciada entre as serragens e a proporção de areia. As densidades seca, úmida e de partícula aumentaram, enquanto o teor de matéria orgânica e a porosidade total foram reduzidos pela adição de areia às serragens. O transplante de miniestacas enraizadas de cacaueiro, clone TSH 1188, para sacos com substrato preparado com serragem coletada no município de Una-BA, nas proporções serragem:areia 4:1 e 2:1, possibilitou maior crescimento das plantas, sendo,portanto, recomendados para produção de mudas.

Termos para indexação: serragem, estaquia, propagação, mudas de cacaueiro

\section{PHYSICAL CHARACTERIZATION OF SAWDUST SUBSTRATE AND CONTAINERS FOR GROWTH CUTTINGS OF CACAO}

\begin{abstract}
The aim of this work was to determine the physical characteristics of substrates of sawdust and to evaluate their effects on the growth of cacao cuttings. The experiment was carried out in random block, $2 \times 4 \times 2$ with factorial design. The treatments were formed from two sawdust residues originated from the districts of Una (SU) and Camacan (SC), Bahia state, Brazil, four proportions (v:v) of sawdust and sand $\left(1: 0 ; 8: 1 ; 4: 1\right.$, and 2:1) and two growth containers (tubettes of $288 \mathrm{~cm}^{3}$ and polyethylene bags of $\left.840 \mathrm{~cm}^{3}\right)$. The Trinidad Select Hybrid clone (TSH 1188) was used for micro-cuttings measuring between 4 and $6 \mathrm{~cm}$ in length. The cuttings were initially treated with IBA $6000 \mathrm{mg} \mathrm{kg}^{-1}$, after inserted in tubes filled with prepared substrates, and then maintained for 40 days in a vapor chamber for root development. Subsequently, the cuttings were transferred to the greenhouse where they either remained in their respective tubes or were transplanted into polyethylene bags and allowed to grow during five months. After this period, the following parameters were evaluated: plant height, stem diameter, above ground biomass and root biomass, number of leaves, and leaf area. Physical analysis of substrates confirmed that particle size distribution was determined by the proportion of sawdust and sand. Dry and wet particle density increased while organic matter content and total porosity decreased with the addition of sand. The transplanting of cuttings in bags used sawdust from Una-BA districts in the proportions sawdust: sand 2:1 and 4:1 facilitated more growth and can be recommending to propagation of cacao cuttings clone TSH 1188.
\end{abstract}

Index terms: sawdust, cacao cuttings, propagation.

\section{INTRODUÇÃO}

A região cacaueira da Bahia sofreu, ao longo da década de 90 , crise econômica que teve como origem a queda dos preços internacionais da commodity cacau. Essa situação foi agravada pelo aparecimento da doença vassoura-de-bruxa (Pereira et al., 1989) causada pelo fungo Crinipellis perniciosa (Stahel) Singer que, no período citado, provocou decréscimo de mais de $60 \%$ na produção de amêndoas secas na região. A resistência genética tem sido a principal ferramenta usada para o controle da doença. Nesse contexto, a produção de mudas de cacaueiros por estaquia tem crescido proporcionalmente à demanda por clones resistentes.

${ }^{1}$ (Trabalho 120-06). Recebido em : 17-08-2006. Aceito para publicação em : 16-03-2007.

${ }^{2}$ Pesquisador da Ceplac/Centro de Pesquisas do Cacau Cepec, C.P. 07, 45600-000 Itabuna, Bahia, Brasil. sodre@cepec.gov.br \& Professor da Universidade Estadual de Santa Cruz Uesc, Departamento de Ciências Agrárias e Ambientais DCAA, km 16, rod. Ilhéus -Itabuna, 45650-000 Ilhéus, Bahia, Brasil.

${ }^{3}$ Professor da Universidade Estadual Paulista Unesp-Campus de Jaboticabal, Departamento de Solos, São Paulo, Brasil,. cora@fcav.unesp.br

${ }^{4}$ Professor da Universidade Estadual de Santa Cruz - Uesc, Departamento de Ciências Agrárias e Ambientais DCAA, km 16, rod. Ilhéus -Itabuna, 45650000 Ilhéus, Bahia, Brasil. olimpio@uesc.br 
Para a certificação das mudas, faz-se necessário usar substratos padronizados, que apresentem características físicas e químicas apropriadas, ausência de patógenos e de sementes de plantas invasoras. Se o custo dos substratos contribui para a elevação do preço final da muda produzida, por outro lado, a utilização de resíduos disponíveis regionalmente pode propiciar redução de custos e minimizar a poluição decorrente do acúmulo desses materiais no ambiente (Fermino, 1996).

$\mathrm{Na}$ região sul do Estado da Bahia, a serragem de madeira é um resíduo que se encontra em serrarias ativas ou desativadas, exposta ao tempo e sem utilidade imediata. $\mathrm{O}$ material apresentase com partículas de diferentes tamanhos, coloração variando de vermelho a marrom e variados graus de umidade e decomposição.

A qualidade da serragem depende do tipo da madeira, do tempo e da condição de armazenamento e do teor de tanino presente (Burés, 1997). Esse autor cita que, dependendo do tempo de armazenamento, a serragem pode ser usada como substrato sem a necessidade de realizar compostagem; entretanto, esse autor ressalta que serragens, ainda que envelhecidas e naturalmente compostadas, podem apresentar fermentação ácida e prejudicar o crescimento das plantas.

De acordo com Burés (1997), substratos com alto percentual de serragem na sua composição podem apresentar problemas de retenção excessiva de umidade. Esse autor recomenda para aumentar a drenagem e reduzir o acúmulo de água que se façam misturas com materiais de maior diâmetro e que apresentem menor capacidade de retenção de água.

Existem registros do uso de areia como componente de substratos para cultivo de cacaueiro multiplicado vegetativamente há mais de meio século, quando Pike (1931) e Fowler (1955) sugeriam o uso de areia e mistura de areia + solo para enraizamento e crescimento de mudas produzidas por estaquia.

A areia é considerada como fração da fase sólida e inorgânica do solo com diâmetro compreendido entre 0,05 e 2 $\mathrm{mm}$. De acordo com o United States Department of Agriculture (USDA), citado por Brady e Weil (2000), a areia pode ser dividida nas classes: areia fina $0,05-0,25 \mathrm{~mm}$ e areia grossa $0,25-2 \mathrm{~mm}$. A densidade da areia varia entre 1.350 e $1.800 \mathrm{~kg} \mathrm{~m}^{-3}$, e a porosidade, que é exclusivamente entre partículas, em geral é inferior a 50\% (Burés, 1997).

As principais vantagens do uso da areia como componente de substratos são: o baixo custo, a estabilidade física e a inatividade química, além da facilidade de limpeza e de tratamento para desinfecção. Por outro lado, os inconvenientes são a alta densidade, que dificulta o manejo, e a baixa retenção de água e nutrientes. Na preparação de substratos, misturas com areia devem especificar a granulometria da areia usada, pois materiais com diferentes tamanhos interferem de forma diferenciada na porosidade e na capacidade de retenção de água das misturas. Para emprego da areia como substrato puro ou em misturas, Martinez (2002) recomenda uma granulometria compreendida entre 0,5 e $2 \mathrm{~mm}$.

Entre os diferentes fatores que afetam o conteúdo de água em substratos, destaca-se o recipiente de cultivo (Fonteno et al.,
1981). Nesse contexto, o tipo de recipiente no qual as mudas de cacaueiro são produzidas, tem influência direta não só na qualidade da muda como também na quantidade de substrato utilizado, mão-de-obra para execução de tratos culturais, espaço ocupado nos viveiros, facilidade de transporte para o campo e custo final da muda.

Este trabalho teve como objetivos realizar caracterização física e avaliar o efeito de substratos à base de serragem no crescimento de mudas obtidas de miniestacas de cacaueiro, em tubetes e sacos de polietileno.

\section{MATERIAL E MÉTODOS}

O experimento foi conduzido nas instalações do Centro de Pesquisas da Comissão Executiva do Plano da Lavoura Cacaueira (Ceplac) em Ilhéus -BA, durante os meses de junho a novembro de 2005. Foi usada a câmara de nebulização para enraizamento de miniestacas e casa de vegetação para crescimento das plantas.

A matéria-prima dos substratos teve como base serragens coletadas em dois locais: municípios de Una e Camacan, no Estado da Bahia, que receberam as denominações de SU e SC, respectivamente. Considerando breve diálogo com moradores dos municípios e o aspecto visual no momento da coleta, essas serragens eram originárias de diferentes espécies da mata Atlântica e diferiam apenas em coloração, sendo a SU vermelha e a SC marrom.

As serragens sofreram processo de compostagem natural durante, pelo menos, 5 anos e encontravam-se em forma de grandes montes ao ar livre. A areia foi coletada em fundo de rio e na análise física apresentou $86 \%$ de areia grossa; $10 \%$ de areia fina; $4 \%$ de argila e densidade de partículas de $2.600 \mathrm{~kg} \mathrm{~m}^{-3}$.

Para retirar a fração grosseira dos materiais, tanto a areia quanto as serragens foram inicialmente passadas em peneira de malha de $6 \mathrm{~mm}$. Para retirada de sais da areia e compostos solúveis das serragens (taninos), ambas foram imersas por 24 horas em água destilada e, posteriormente, secas à sombra.

O experimento foi constituído por fatorial $2 \times 4 \times 2$. Os tratamentos foram formados pela origem das serragens ( $\mathrm{SU}$ e SC), quatro proporções (v:v) de serragem e areia: 1:0; 8:1; 4:1 ; 2:1 e dois recipientes de crescimento (tubetes de $288 \mathrm{~cm}^{3}$ e sacos de polietileno de $840 \mathrm{~cm}^{3}$ ). O delineamento foi em blocos casualizados, com três repetições e três plantas por parcela, sendo colocada 1 planta (miniestaca enraizada) por recipiente.

Inicialmente, os substratos foram caracterizados quanto à granulometria em peneiras de 0,$25 ; 0,50 ; 1,0 ; 2,0$ e $4,0 \mathrm{~mm}$ e determinadas as densidades úmida (DU) e seca (DS), segundo Hoffman, descrito por Fermino (1996); a densidade de partículas (DP) e o teor de matéria orgânica (MO), segundo Ansorena (1994), e a porosidade total (PT), conforme De Boodt e Verdonck (1972).

Para a produção das mudas, foram usadas miniestacas de ramos plagiotrópicos do clone Trinidad Select Hybrid (TSH 1188). As plantas-matrizes encontravam-se em viveiro telado na Ceplac, vegetando há cinco anos, numa mistura de fibra de coco e casca de Pinus 1:1 (v:v), em sacos de polietileno de $22 \mathrm{dm}^{3}$. 
As miniestacas foram retiradas da ponta dos ramos das plantas-matrizes e tiveram comprimento final de 4 a $6 \mathrm{~cm}$. A base da miniestaca foi cortada transversalmente, $2 \mathrm{~mm}$ abaixo de uma gema foliar e, em seguida, a primeira folha da base para o ápice foi reduzida à metade, e as demais, em $20 \%$ do tamanho original.

Visando a homogeneizar a umidade dos substratos, antes do estaqueamento, os tubetes de $288 \mathrm{~cm}^{3}$, depois de preenchidos com as combinações de serragem e areia, permaneceram na câmara de nebulização por 48 horas, recebendo nebulização por 15 segundos a cada 5 minutos.

As miniestacas foram inicialmente tratadas na base com ácido indolbutírico (AIB) misturado em talco $6.000 \mathrm{mg} \mathrm{kg}^{-1} \mathrm{e}, \mathrm{em}$ seguida, inseridas nos tubetes a $1,5 \mathrm{~cm}$ de profundidade, nos devidos tratamentos. Para manter a atmosfera saturada a $100 \%$ de umidade relativa na superfície da folha, as miniestacas foram submetidas a nebulização por 15 segundos, a cada 5 minutos, entre as 6 e as $18 \mathrm{hs}$, e 15 segundos, a cada hora, das 18 às 6 horas do dia seguinte.

Quando se verificou a presença de raízes primárias e o início das primeiras brotações, o que ocorreu aos quarenta dias, as miniestacas foram retiradas da câmara de nebulização e transferidas para crescimento em casa de vegetação, onde parte foi mantida nos respectivos tubetes e outra transplantada em sacos de polietileno de $840 \mathrm{~cm}^{3}$, preenchidos com os mesmos substratos.

As aplicações de fertilizantes foram realizadas de acordo com Marrocos e Sodré (2004). Na primeira semana do período de crescimento, foram adicionados aos tubetes e sacos, $2,0 \mathrm{~g} \mathrm{dm}^{-3}$ do fertilizante de liberação lenta (3-5 meses) Osmocote ${ }^{\circledR}(22 \% \mathrm{~N}$ - $04 \% \mathrm{P}_{2} \mathrm{O}_{5}-08 \% \mathrm{~K}_{2} \mathrm{O}$ ) e $1,0 \mathrm{~g} \mathrm{dm}^{-3}$ do fertilizante solúvel PG Mix $14 \% \mathrm{~N}^{2}-18 \% \mathrm{P}_{2} \mathrm{O}_{5}-18 \% \mathrm{~K}_{2} \mathrm{O}+$ micronutrientes. Durante o crescimento, as mudas receberam, diariamente, irrigações por microaspersão com duração de três minutos a cada três horas.

Após cinco meses de permanência das mudas em casa de vegetação, avaliaram-se: altura da planta (AP), diâmetro do caule (DC), massa da matéria seca da parte aérea (MSPA) e das raízes (MSR), número de folhas (NF) e área foliar (AF). Os dados foram submetidos à análise de variância, usando-se o programa SAS ${ }$ (SAS, 1989) e as médias foram comparadas pelo teste de Tukey, a $5 \%$ de probabilidade.

\section{RESULTADOS E DISCUSSÃO}

\section{Análises físicas dos substratos}

A distribuição do tamanho das partículas dos substratos variou com a origem da serragem e a proporção de areia (Tabela 1). A adição de areia à serragem do município de Una $S U$ aumentou a porcentagem em massa de partículas de diâmetro entre 2-0,5 mm. Para os demais diâmetros, a adição de areia reduziu a porcentagem em massa.

As serragens apresentaram maior porcentagem em massa para o diâmetro de $1-0,5 \mathrm{~mm}$, seguido de $0,5-0,25 \mathrm{~mm}$, contudo os resultados da serragem do município de Camacan SC não apresentaram o mesmo padrão da SU. A diferença observada entre as serragens discorda do conceito elaborado por Loures
(1983), que classifica a serragem como material uniforme e homogêneo.

A SU na proporção serragem:areia 1:0 diferenciou-se dos demais substratos por apresentar maior percentual $(22,2 \%)$ da fração menor que $0,25 \mathrm{~mm}$ (Tabela 1). Essa característica classifica a SU 1:0 como substrato que apresenta alta porcentagem de partículas finas e que, segundo Fernandes (2005), tende a aumentar a retenção de água, diminuindo a disponibilidade para as plantas. Outra conseqüência é a diminuição na aeração. Burés (1997) ressaltou que o principal problema do uso da serragem com elevada quantidade de partículas finas é o risco de compactação que reduz a aeração, podendo ocorrer processos anaeróbios de fermentação, gerando ácidos orgânicos que interferem no crescimento de raízes.

Outras características dos substratos são apresentadas na Tabela 2. Observa-se que a DU, DS e DP aumentaram com adição de areia. Resultados de aumento de densidade em substratos à base de turfa e cascas de amendoim, pela adição de areia, foram respectivamente citados por Martinez (2002) e encontrados por Fernandes (2005).

O teor de matéria orgânica (MO) e a porosidade total (PT) foram reduzidos pela adição de areia. Resultados semelhantes foram verificados por Bunt (1983) e Handreck (1983) em substratos à base de turfa e casca de Pinus. A redução da porosidade pela adição de areia ocorre, segundo Burés (1997), porque a porosidade de substratos depende mais do estado de empacotamento das partículas, que resulta do tamanho dessas e de como se encontram distribuídas, do que da granulometria individual de cada componente do substrato.

\section{Características do crescimento das mudas}

Por se tratar de experimento com três fatores (origem da serragem, recipiente e proporção de areia), optou-se por apresentar os resultados da análise da variância e discutir as interações significativas, que são mais relevantes que os efeitos principais. Foram verificadas, para o DC, interações entre o fator origem e recipiente, proporção de areia e recipiente e também interação tripla entre serragem, areia e recipiente (Tabela 3 ).

Verificou-se, independentemente da origem da serragem, que o diâmetro do caule (DC) e a MSPA das plantas crescidas em sacos foi superior aos tubetes. Para as plantas crescidas em tubetes, não houve diferença significativa para o DC e MSPA em resposta à serragem utilizada. No entanto, o crescimento das plantas, em sacos de polietileno, foi superior para SU quando comparado a SC (Tabela 4).

Resultados semelhantes em experimento com recipientes foram encontrados por Mendonça et al. (2003) na cultura de mamoeiro. Esses autores verificaram aumentos significativos na MSPA de mudas crescidas em sacos de $0,75 \mathrm{dm}^{3}$, quando comparado a tubetes de $0,05 \mathrm{dm}^{3}$ e também atribuíram a menor resposta das plantas crescidas em tubetes à limitação por nutrientes.

Alterações na capacidade de recipiente podem, segundo White \& Mastalerz (1966), alterar o gradiente de distribuição de umidade e aeração determinando crescimento diferenciado das 
plantas. Assim, no presente trabalho, os diferentes volumes de substrato disponíveis para as plantas, possivelmente, determinaram diferentes capacidades de recipiente, o que explicaria a superioridade das plantas crescidas em sacos de polietileno.

Quando se analisou a proporção de areia dentro de cada tipo de recipiente, verificaram-se maiores médias das plantas em DC quando transplantadas para sacos (Tabela 5). Verificou-se também que a redução da porosidade total pela adição de areia, apresentada na Tabela 2, não interferiu no DC das plantas, que foi significativamente superior na proporção 2:1 e 4:1 em relação à 1:0, respectivamente, para tubetes e sacos (Tabela 5). Esse resultado é contrário ao esperado para um bom meio de cultivo, que De Boodt e Verdonck (1972) recomendam seja superior a $0,85 \mathrm{~m}^{3} \mathrm{~m}^{-3}$. Contudo, Fermino (2002) e Martinez (2002) relataram que, quando se mistura areia de alta granulometria em baixas e médias proporções, não se melhora a aeração ou a porosidade de substratos. Por outro lado, Fonteno et al. (1981) e Drzal et al. (1999) verificaram que a porosidade e o espaço de aeração são fortemente alterados pelo modo de enchimento do recipiente, transporte e acomodação dos substratos. Assim, valores encontrados antes do enchimento dos recipientes foram, possivelmente, alterados durante o cultivo, fazendo com que plantas crescendo em substratos com porosidade inicial baixa, apresentassem crescimento superior àquelas com maior porosidade.

TABELA 1 - Distribuição do tamanho de partículas de substratos à base de serragem e areia usados no enraizamento $\mathrm{e}$ crescimento de mudas de cacaueiro Ilhéus-BA, 2005.

\begin{tabular}{|c|c|c|c|c|c|c|c|}
\hline \multirow[t]{2}{*}{$\begin{array}{l}\text { Local de origem da } \\
\text { serragem }\end{array}$} & \multirow[t]{2}{*}{$\begin{array}{c}\text { Proporção } \\
\text { serragem : areia } \\
\text { (v:v) }\end{array}$} & \multicolumn{6}{|c|}{ Classes de diâmetro das partículas (mm) } \\
\hline & & $>4$ & $4-2$ & $2-1$ & $1-0,5$ & $0,5-0,25$ & $<0,25$ \\
\hline & & & & $\mathrm{em} \mathrm{ma}$ & & & \\
\hline \multirow{4}{*}{ Una } & 1:0 & 0,9 & 4,2 & 8,6 & 33,8 & 30,3 & 22,2 \\
\hline & $8: 1$ & 0,7 & 4,1 & 12,9 & 43,6 & 27,9 & 10,8 \\
\hline & 4:1 & 0,3 & 3,5 & 12,0 & 46,4 & 28,8 & 9,0 \\
\hline & $2: 1$ & 0,3 & 1,3 & 11,8 & 47,7 & 30,0 & 8,9 \\
\hline \multirow{4}{*}{ Camacan } & 1:0 & 0,3 & 3,7 & 10,9 & 40,2 & 25,6 & 19,3 \\
\hline & $8: 1$ & 1,2 & 8,4 & 13,8 & 46,5 & 21,2 & 8,9 \\
\hline & $4: 1$ & 0,8 & 2,4 & 11,3 & 44,9 & 28,5 & 12,1 \\
\hline & $2: 1$ & 0,6 & 1,6 & 10,4 & 49,4 & 27,4 & 10,6 \\
\hline
\end{tabular}

Considerando o efeito da proporção de areia na origem da serragem, o DC das plantas crescidas na proporção de areia 4:1 da SU foi superior a 1:0 e 8:1 e não diferiu estatisticamente da $2: 1$. Foi também observado que diferenças entre as serragens ocorreram com o aumento da proporção de areia (Tabela 6).

$\mathrm{O}$ alto percentual da fração $<0,25 \mathrm{~mm}$, destacadamente na SU 1:0 (Tabela 1), pode ter contribuído para aumentar o empacotamento desse substrato, encher os espaços vazios, reduzir a aeração e, conseqüentemente, influenciar no crescimento do DC. Estas observações estão de acordo com os relatos de Mourão Filho et al. (1998), que também verificaram aumentos significativos em altura e diâmetro de porta-enxerto de Citrumeleiro "Swingle" utilizando substrato composto de terra, raspa de madeira, esterco bovino e areia, na proporção de $25 \%$ para cada componente, em relação ao tratamento com $100 \%$ de terra.

Devido à não-ocorrência de diferenças significativas no DC das plantas crescidas na SU, nas proporções 4:1 e 2:1 (Tabela 6), a proporção 4:1, por ser mais leve, apresenta-se como a melhor opção para otimizar o uso desta serragem. Isso porque o peso dificulta o manuseio das mudas, especialmente quando transportadas por homens ou animais para o local de plantio, prática comum na região cacaueira do Estado da Bahia. Essa observação é corroborada por Martinez (2002), que relata ser o peso a principal limitação para uso e transporte de substratos que contenham areia.

TABELA 2 - Características ${ }^{1}$ de substratos à base de serragem e areia usados no enraizamento e crescimento de mudas de cacaueiro.

\begin{tabular}{lcccccc}
\hline $\begin{array}{c}\text { Local de origem } \\
\text { da serragem }\end{array}$ & $\begin{array}{c}\text { Proporção } \\
\text { serragem: areia } \\
(\mathrm{v}: \mathrm{v})\end{array}$ & $\mathrm{DU}$ & $\mathrm{DS}$ & $\mathrm{MO}$ & $\mathrm{DP}$ & $\mathrm{PT}$ \\
\hline \multirow{3}{*}{ Una } & $1: 0$ & 730 & 240 & 888 & 1360 & 0,83 \\
& $8: 1$ & 780 & 360 & 499 & 1880 & 0,83 \\
& $4: 1$ & 870 & 460 & 340 & 2160 & 0,72 \\
& $2: 1$ & 990 & 650 & 101 & 2500 & 0,74 \\
\hline \multirow{3}{*}{ Camacan } & $1: 0$ & 550 & 210 & 706 & 1360 & 0,93 \\
& $8: 1$ & 670 & 280 & 537 & 1870 & 0,84 \\
& $4: 1$ & 800 & 430 & 295 & 2220 & 0,83 \\
& $2: 1$ & 920 & 620 & 181 & 2390 & 0,77 \\
\hline
\end{tabular}

${ }^{1}$ DU: Densidade úmida; DS: Densidade seca; MO: Matéria orgânica; DP: Densidade de partícula; PT: Porosidade total.

TABELA 3 - Valores dos quadrados médios obtidos na análise de variância para efeito de dois locais de origem de serragem ${ }^{1}$, dois recipientes ${ }^{2}$ e quatro proporções de serragem: areia $^{3}$ utilizados no crescimento de mudas de cacaueiro ${ }^{4}$.Ilhéus-BA,2005.

\begin{tabular}{|c|c|c|c|c|c|c|c|c|}
\hline \multirow[b]{2}{*}{ Causas da variação } & \multirow[b]{2}{*}{ GL } & \multicolumn{7}{|c|}{ Quadrados Médios } \\
\hline & & AP & DC & MSPA & MSR & AF & NF & AF/NF \\
\hline Blocos & 2 & $1,010^{\mathrm{ns}}$ & $0,020^{n s}$ & $0,075^{n s}$ & $0,037^{n s}$ & $1.527,918^{\mathrm{ns}}$ & $1,538^{\mathrm{ns}}$ & $82,009^{\mathrm{ns}}$ \\
\hline $\begin{array}{c}\text { Local de origem da } \\
\text { serragem (O) }\end{array}$ & 1 & $235,021 * * *$ & $1,803 * * *$ & $5,867 * * *$ & $0,018^{n s}$ & $288.216,668 * * *$ & $26.462 * *$ & $556,557 * * *$ \\
\hline Areia (A) & 3 & $31,516 *$ & $0,406 * * *$ & $0,507 *$ & $0,016^{\mathrm{ns}}$ & $17.491,015^{\mathrm{ns}}$ & $1,171 \mathrm{~ns}$ & $88,134^{\mathrm{ns}}$ \\
\hline Recipiente ( $R$ ) & 1 & $2.316,607 * * *$ & $22,534 * * *$ & $47,859 * * *$ & $1,136 * * *$ & $3.868 .147,297 * * *$ & $296,697 * * *$ & $14.592,150 * *$ \\
\hline$O \times A$ & 3 & $23,510^{\mathrm{ns}}$ & $0,159^{\mathrm{ns}}$ & $0,366^{\mathrm{ns}}$ & $0,050^{\mathrm{ns}}$ & $19.930,702^{\mathrm{ns}}$ & $1,242^{\mathrm{ns}}$ & $105,373^{\mathrm{ns}}$ \\
\hline$O \times R$ & 1 & $9,268^{\mathrm{ns}}$ & $1,439 * * *$ & $1,162 * \cdots *$ & $0,055^{\mathrm{ns}}$ & $37.207,134^{\mathrm{ns}}$ & $1,811 \mathrm{~ns}$ & $43,001 \mathrm{~ns}$ \\
\hline$A \times R$ & 3 & $8,932^{\mathrm{ns}}$ & $0,340 *$ & $0,178^{\mathrm{ns}}$ & 0,031 ns & $5.334,032^{\mathrm{ns}}$ & $2,662^{\mathrm{ns}}$ & $18,402^{n s}$ \\
\hline$O \times A \times R$ & 3 & $2,164^{n s}$ & $0,651 * * *$ & $0,377^{\mathrm{ns}}$ & $0,045^{\mathrm{ns}}$ & $4.799,872^{\mathrm{ns}}$ & $2,496 \mathrm{~ns}$ & $46,515^{\mathrm{ns}}$ \\
\hline Resíduo & 32 & 9,016 & 0,0861 & 0,162 & 0,020 & $15.075,310$ & 2,471 & 35,315 \\
\hline CV (\%) & & 15,0 & 7,4 & 19,6 & 28,1 & 23,7 & 19,0 & 11,0 \\
\hline
\end{tabular}

${ }^{1}$ Local de origem das serragens-municípios: Una (SU) e Camacan (SC). ${ }^{2}$ Recipientes: saco de polietileno de $840 \mathrm{~cm}^{3}$ e tubete de $288 \mathrm{~cm}{ }^{3}{ }^{3}$ Proporções serragem e areia: 1:0, 8:1, 4:1 e 2:1 (v/v). ${ }^{4}$ Altura da planta (AP), diâmetro do caule (DC), massa da matéria seca da parte aérea (MSPA) e das raízes (MSR), área foliar (AF), número de folhas $(\mathrm{NF})$ e relação $(\mathrm{AF} / \mathrm{NF})$. ns: não- significativo *, **, ***: significativos a 5,$0 ; 1,0$ e $0,1 \%$ de probabilidade. CV (\%): coeficiente de variação. 
TABELA 4 - Efeito de cada nível de serragem dentro do tipo de recipiente para o diâmetro do caule e massa da matéria seca de parte aérea de mudas de cacaueiro. Ilhéus-BA, 2005.

\begin{tabular}{|c|c|c|c|c|}
\hline \multirow[t]{3}{*}{ Local de origem da serragem } & \multicolumn{4}{|c|}{ Recipientes } \\
\hline & Tubete & Saco & Tubete & Saco \\
\hline & \multicolumn{2}{|c|}{ Diâmetro do caule } & \multicolumn{2}{|c|}{ Massa da matéria seca da parte aérea } \\
\hline Una & $0,32^{(1)} \mathrm{aB}$ & $0,50 \mathrm{aA}$ & $1,19 \mathrm{aB}$ & $3,59 \mathrm{aA}$ \\
\hline Camacan & $0,32 \mathrm{aB}$ & $0,42 \mathrm{bA}$ & $0,92 \mathrm{aB}$ & $2,54 \mathrm{bA}$ \\
\hline
\end{tabular}

${ }^{1}$ Médias seguidas pela mesma letra minúscula na coluna (entre serragens) e maiúscula na linha (entre recipientes) não diferem entre si, pelo teste de Tukey, a $5 \%$ de probabilidade.

TABELA 5 - Efeito da proporção entre serragem e areia dentro de cada tipo de recipiente para o diâmetro do caule de mudas de cacaueiro. Ilhéus-BA, 2005.

\begin{tabular}{|c|c|c|}
\hline \multirow{3}{*}{ Proporção serragem : areia (v:v) } & \multicolumn{2}{|c|}{ Recipiente } \\
\hline & Tubete & Saco \\
\hline & \multicolumn{2}{|c|}{ Diâmetro do caule } \\
\hline $1: 0$ & $0,29^{(1)} \mathrm{bB}$ & $0,45 \mathrm{bA}$ \\
\hline $8: 1$ & 0,32 abB & $0,44 \mathrm{bA}$ \\
\hline $4: 1$ & 0,33 abB & $0,50 \mathrm{aA}$ \\
\hline $2: 1$ & $0,35 \quad \mathrm{aB}$ & $0,45 \mathrm{bA}$ \\
\hline
\end{tabular}

${ }^{1}$ Médias seguidas pela mesma letra minúscula na coluna e maiúscula na linha não diferem entre si, pelo teste de Tukey, a 5\% de probabilidade.

TABELA 6 - Efeito da proporção serragem e areia dentro de cada local de origem da serragem para o diâmetro do caule de mudas de cacaueiro. Ilhéus-BA, 2005

\begin{tabular}{|c|c|c|}
\hline & \multicolumn{2}{|c|}{ Local de origem da serragem } \\
\hline \multirow[t]{2}{*}{ Proporção serragem : areia (v:v) } & Una & Camacan \\
\hline & \multicolumn{2}{|c|}{ Diâmetro do caule } \\
\hline $1: 0$ & $0,38^{(1)} \mathrm{bA}$ & $0,36 \mathrm{aA}$ \\
\hline $8: 1$ & $0,39 \quad \mathrm{bA}$ & $0,37 \mathrm{aA}$ \\
\hline $4: 1$ & 0,44 aA & $0,38 \mathrm{aB}$ \\
\hline $2: 1$ & $0,42 \quad a b A$ & $0,38 \mathrm{aB}$ \\
\hline
\end{tabular}

${ }^{1}$ Médias seguidas pela mesma letra minúscula na coluna e maiúscula na linha não diferem entre si, pelo teste de Tukey, a 5\% de probabilidade

\section{CONCLUSÃO}

O transplante de miniestacas enraizadas de cacaueiro, clone TSH 1188, para sacos com substrato preparado com serragem coletada no município de Una-BA, nas proporções serragem:areia 4:1 e $2: 1$, possibilitou maior crescimento das plantas, sendo, portanto, recomendados para produção de mudas.

\section{AGRADECIMENTOS}

Os autores agradecem ao técnico agrícola Edmundo Andrade e ao analista de sistemas Renato Novaes, pelas importantes colaborações durante a condução e análise do experimento.

\section{REFERÊNCIAS}

ANSORENA, J.M. Sustratos: propiedades y caracterización. Madrid: Mundi-Prensa, 1994. 172p.

BRADY, N.C.; WEIL, R.R. Elements of the nature and properties of soils. $12^{\text {th }}$ ed. New Jersey: Prentice-Hall, 2000. 559 p.

BUNT, A. C. Physical properties of mixtures of peat and minerals of different particle size and bulk density for potting substrates. Acta Horticulturae, Wageningen, v. 150, p.143$153,1983$.

BURÉS, S. Sustratos. Madri: Agrotécnicas, 1997. 342 p.

De BOODT, M; VERDONCK. The physical properties of the substrates in horticulture. Acta Horticulturae, Wageningen, v. 26, p.37-44, 1972.

DRZAL, M.S.; CASSEL,D.K.; FONTENO,W.C. Pore fraction analysis: a new tool for substrate testing. Acta Horticulturae, Wageningen, v.1, n.481, p.43-53, 1999.

FERMINO, M.H. Aproveitamento de resíduos industriais e agrícolas como alternativas de substratos Hortícolas. 1996. 90 f. Dissertação (Mestrado em Fitotecnia) - Faculdade de Agronomia, Universidade Federal do Rio Grande do Sul, 1996.

FERMINO, M.H. Uso da análise física na avaliação da qualidade de componentes de substratos. In: ENCONTRO NACIONAL SOBRE SUBSTRATO PARA PLANTAS, 3., 2002, Campinas.

FONTENO, W.C.; CASSEL,D.K.; LARSON,R.A. Physical properties of three container media and their effect on poinsettia growth. Journal of American Society for Horticultural Science, Alexandria, v.106, n. 6, p.736-741, 1981.

FERNANDES, C. Produtividade e qualidade dos frutos do tomateiro do grupo cereja cultivado em substratos à base de areia. 2005. 85f. Tese (Doutorado em Produção Vegetal) Faculdade de Ciências Agrárias e Veterinárias, Universidade Estadual Paulista, Jaboticabal, 2005.

FOWLER, R. L. Propagação de cacau por meio de estacas. Santa Catarina: Escritório técnico de Agricultura- ETA Brasil Estados Unidos, 1955. (Avulso IL, 3)

HANDRECK,K.A. Particle-size and the physical-properties of growing media from containers. Communications in soil science and plant analysis, New York, v. 14, n. 3, p. 209-222, 1983.

LOURES, E. G. Produção de composto no meio rural. Viçosa : UFLA, 1983. 12p. (Informe Técnico, 17)

MARROCOS, P.C.L.; SODRÉ, G.A. Sistema de Produção de mudas de cacaueiros. In: ENCONTRO NACIONAL SOBRE SUBSTRATOS PARA PLANTAS, 4., 2004, Viçosa.

MARTINEZ, P. F. Manejo de sustratos para horticultura. In: Encontro nacional sobre substrato para plantas . . In: ENCONTRO NACIONAL SOBRE SUBSTRATOS PARA 
PLANTAS, 3., 2002, Campinas.

MENDONÇA, V.; ARAÚJO NETO, S.E.; RAMOS,J.D.; PIO,R.;

GONTIJO,T.C.A. Diferentes substratos e recipientes na formação de mudas de mamoeiro "Sunrise Solo". Revista Brasileira de Fruticultura, Jaboticabal, v. 25, n.1, p.127130, 2003.

MOURÃO, Filho.A.A.; DIAS,C.T.S.; SALIBE.A.A. Efeito da composição do substrato na formação de mudas de laranjeira 'Pêra'. Scientia Agricola, Piracicaba, v. 55, n.1, p. 35-42, 1998.

PEREIRA,J.L.; Ram, A.; FIGUEIREDO,J.M.; ALMEIDA,L.C. Primeira ocorrência de vassoura-de-bruxa na principal região produtora de cacau do Brasil. Agrotrópica, Ilhéus, v.1, n.1, p.79-81, 1989.

PYKE, E. E. The vegetative propagation of Theobroma cacao by softwood cuttings. Tropical Agriculture, Trinidad, v. 8, n. 9, p. 249, 1931.

SAS INSTITUTE. SAS/STAT user's guide: version $6.4^{\text {th }} \mathrm{ed}$. Cary, 1989. v.2. 1686 p.

WHITE, J.W.; MASTALERZ, J.W. Soil moisture as related to "Container Capacity" Proceedings of the American Society for Horticultural Science, Beltsville, v. 89, n.1, p. 758-765, 1966. 